\title{
Famille, communauté et folie au tournant du siècle
}

\section{Thierry Nootens}

Volume 53, numéro 1, été 1999

Médecine, santé et sociétés

URI : https://id.erudit.org/iderudit/005555ar

DOI : https://doi.org/10.7202/005555ar

Aller au sommaire du numéro

\section{Éditeur(s)}

Institut d'histoire de l'Amérique française

\section{ISSN}

0035-2357 (imprimé)

1492-1383 (numérique)

Découvrir la revue

\section{Citer cet article}

Nootens, T. (1999). Famille, communauté et folie au tournant du siècle. Revue d'histoire de l'Amérique française, 53(1), 93-119.

https://doi.org/10.7202/005555ar
Résumé de l'article

RÉSUMÉ

Voulant contribuer à l'histoire de la « folie " au Québec, cet article met à profit les riches possibilités offertes par des archives judiciaires, les dossiers d'interdiction et de curatelle, pour souligner le rôle de l'instance familiale et communautaire dans la prise en charge et l'exclusion de la maladie mentale au tournant du siècle. À l'aide des mesures réglant l'incapacité de majeurs considérés mentalement déviants, on peut identifier quelques données importantes de l'expérience de l'aliénation mentale de plusieurs dizaines d'individus des Cantons de l'Est. Des particularités sociologiques ou juridiques pouvaient influencer le passage d'une personne devant la justice civile, et l'examen des infractions aux normes dénoncées dans les dossiers permet de cerner les préoccupations des membres de la famille et de l'entourage. Ceux-ci, placés en première ligne, devaient recourir à des stratégies particulières, $\mathrm{y}$ compris l'appel à la justice, pour gérer la conduite d'un individu aux comportements hors normes. Enfin, même si on faisait largement appel à l'avis des médecins et des aliénistes lors des procès, l'expertise des médecins de la région, en ce qui a trait à la maladie mentale, paraît douteuse. L'analyse conduit à remettre en question l'utilité générale de la perspective voulant que la réponse à la folie ait été essentiellement, au XIXe et au début du XXe siècles, une entreprise institutionnelle de contrôle social menée par les élites bourgeoises, médicales et étatiques.
Tous droits réservés @ Institut d'histoire de l'Amérique française, 1999
Ce document est protégé par la loi sur le droit d'auteur. L'utilisation des services d'Érudit (y compris la reproduction) est assujettie à sa politique d'utilisation que vous pouvez consulter en ligne.

https://apropos.erudit.org/fr/usagers/politique-dutilisation/ 


\title{
FAMILLE, COMMUNAUTÉ ET FOLIE AU TOURNANT DU SIÈCLE
}

\author{
THIERRY NOOTENS \\ Département d'histoire \\ Université du Québec à Montréal
}

\begin{abstract}
RÉSUMÉ
Voulant contribuer à l'histoire de la «folie » au Québec, cet article met à profit les riches possibilités offertes par des archives judiciaires, les dossiers d'interdiction et de curatelle, pour souligner le rôle de l'instance familiale et communautaire dans la prise en charge et l'exclusion de la maladie mentale au tournant du siècle. À l'aide des mesures réglant l'incapacité de majeurs considérés mentalement déviants, on peut identifier quelques données importantes de l'expérience de l'aliénation mentale de plusieurs dizaines d'individus des Cantons de l'Est. Des particularités sociologiques ou juridiques pouvaient influencer le passage d'une personne devant la justice civile, et l'examen des infractions aux normes dénoncées dans les dossiers permet de cerner les préoccupations des membres de la famille et de l'entourage. Ceux-ci, placés en première ligne, devaient recourir à des stratégies particulières, y compris l'appel à la justice, pour gérer la conduite d'un individu aux comportements hors normes. Enfin, même si on faisait largement appel à l'avis des médecins et des aliénistes lors des procès, l'expertise des médecins de la région, en ce qui a trait à la maladie mentale, paraît douteuse. L'analyse conduit à remettre en question l'utilité générale de la perspective voulant que la réponse à la folie ait été essentiellement, au $\mathrm{XIX}^{\mathrm{e}}$ et au début du $\mathrm{XX}^{\mathrm{e}}$ siècles, une entreprise institutionnelle de contrôle social menée par les élites bourgeoises, médicales et étatiques.
\end{abstract}

\section{ABSTRACT}

This article makes use of judicial archives (interdiction and curatorship cases) to examine the role of family and community in the care and exclusion of mental illness at the turn of the century. The measures regulating the legal incapacity of mentally disabled adults reveal important aspects of the experience of mental alienation by Eastern-Townships inhabitants. Sociological or judicial particularities could influence the citation of a person before the court; the misdemeanours reported in the files, in particular, help establish the concerns of those relatives, friends and neighbours, whom propinquity forced to resort to specific strategies for managing deviant behavior. Finally, though doctors and alienists were often called upon in such in cases, local physicians' expertise seems particularly dubious. Overall, this article questions views that cast the late-nineteenth and early-twentieth-century responses to insanity as institutional exercises in social control driven by state and medical bourgeois elites. 
Une histoire sociale de la folie ${ }^{1}$, c'est-à-dire un discours historique dépassant le simple récit des événements pour s'interroger sur les liens entre folie et société, vit le jour au Québec dans les années 1970². Parus peu après l'article fondateur de Fernand Harvey $^{3}$ et la courte monographie institutionnelle signée Céline Beaudet ${ }^{4}$, les Essais pour une préhistoire de la psychiatrie au Canada (1800-1885) ${ }^{5}$, du groupe dirigé par André Paradis, inaugurèrent véritablement la recherche par une réflexion portant essentiellement sur l'asile et ses raisons d'apparition au XIX ${ }^{\mathrm{e}}$ siècle.

Les quinze dernières années ont connu une diversification des problèmes traités et des cadres d'analyse mis en œuvre. Les travaux de Guy Boisclair et d'André Cellard ${ }^{6}$, par leur intérêt envers la question des représentations de la folie et des attitudes entourant ce phénomène, illustrent bien une approche relevant de l'histoire des mentalités. L'histoire de la psychiatrie en tant que connaissance peut, quant à elle, compter sur l'ouvrage de Peter Keating, La science du mal, et sur le mémoire de Guy Grenier ${ }^{7}$. Sur le plan juridique, Louise Labrèche-Renaud s'est intéressée au sort de nombreux accusés reconnus malades mentaux dans la seconde moitié du XIX ${ }^{\mathrm{e}}$ siècle et au rôle joué par les asiles envers ces gens ${ }^{8}$. En adoptant une perspective sociologique, l'ouvrage de Françoise Boudreau et le mémoire de Daniel Bouchard et Simon Doucet ${ }^{9}$ ont privilégié l'exa-

1. Les recherches de maîtrise ayant mené à la réalisation du présent article ont été soutenues par le Fonds FCAR, organisme auquel l'auteur témoigne la plus vive reconnaissance, de même qu'à son directeur de recherche d'alors, le professeur Peter Gossage. Merci également à Jean-Marie Fecteau et aux deux évaluateurs externes de la Revue d'histoire de l'Amérique française pour leurs judicieuses critiques.

2. On trouvera une recension complète de l'historiographie de la folie au Québec dans Thierry Nootens, To Be Quiet, Orderly, Obedient and Industrious : la normalité dans le district judiciaire de Saint-François entre 1880 et 1920 d'après l'interdiction des « malades mentaux », mémoire de maîtrise, Université de Sherbrooke, 1997, 159 p.

3. Fernand Harvey, «Préliminaires à une sociologie historique des maladies mentales au Québec », Recherches sociographiques, 16,1 (janvier 1975) : 113-117.

4. Céline Beaudet, Évolution de la psychiatrie anglophone au Québec : 1880-1963. Le cas de l'hôpital de Verdun (Québec, Institut supérieur des sciences humaines de l'Université Laval, 1976), 126 p.

5. André Paradis, dir., et al., Essais pour une préhistoire de la psychiatrie au Canada (1800-1885) (Trois-Rivières, Département de philosophie de l'Université du Québec à Trois-Rivières, 1977), 345 p.

6. Guy Boisclair, La perception de la folie au Québec au XVIII siècle, mémoire de maîtrise, Université de Sherbrooke, 1989, 183 p.; André Cellard, Histoire de la folie au Québec de 1600 à 1850. Le désordre (Montréal, Boréal, 1991), 280 p.

7. Peter Keating, La science du mal. L'institution de la psychiatrie au Québec 1800-1914 (Montréal, Boréal, 1993), 208 p.; Guy Grenier, L'implantation et les applications de la doctrine de la dégénérescence dans le champ de la médecine et de l'hygiène mentale au Québec entre 1885 et 1930, mémoire de maîtrise, Université de Montréal, 1990, 172 p.

8. Louise Labrèche-Renaud, Les racines juridiques de l'aliénation mentale et l'institutionnalisation au Québec, de 1845 à 1892, thèse (LL.D.), Université de Montréal, 1991, 570 p.

9. Françoise Boudreau, De l'asile à la santé mentale. Les soins psychiatriques : histoire et institutions (Montréal, Éditions Saint-Martin, 1984), 274 p.; Daniel Bouchard et Simon Doucet, 
men des rapports de pouvoir entre les divers groupes d'intérêts historiquement concernés par la psychiatrie.

Malgré ces progrès, l'histoire de la folie au Québec ne bénéficie pas encore d'une analyse précise de ce que pouvait représenter, à une époque ou à une autre, la présence de l'aliénation mentale dans la communauté. Les archives de l'interdiction permettent de reconstituer quelques-unes des grandes lignes de la « gestion » de personnes ayant subi un ostracisme juridique parce que considérées mentalement malades. Nous avons pris pour cible les individus interdits entre 1880 et 1920 dans le district judiciaire de Saint-François (Cantons de l'Est). Les nombreux procès viennent éclairer la façon dont était vécue l'irruption de troubles mentaux ou de comportements supposés tels. Ils conduisent de plus à relativiser certaines thèses centrales de l'histoire de la folie, dont celle voulant que la réponse à ce phénomène ait été, au XIX ${ }^{\mathrm{e}}$ siècle, d'abord et avant tout une entreprise institutionnelle de contrôle social menée par les élites, qu'elles soient bourgeoises, médicales (la folie aurait subi un processus de médicalisation) ou étatiques ${ }^{10}$. Dans ce cadre, l'industrialisation, l'urbanisation accélérée et la transition au capitalisme des sociétés occidentales sont souvent évoquées pour expliquer le bouleversement majeur que représente l'avènement de l'asile.

L'analyse des interdictions porte à nuancer cette perspective et surtout à souligner le rôle central de la famille et de la communauté dans la prise en charge de la folie et ce, même après la mise en place d'un imposant réseau asilaire au Québec. On peut alors appréhender une dynamique sociale beaucoup plus complexe où la famille, et non les élites, domine; où l'industriali-

L'État et l'administration des institutions asilaires au Québec : 1845-1895, mémoire de maîtrise (histoire), Université du Québec à Montréal, 1985, 216 p. André Paradis a mis en lumière la donnée la plus essentielle de l'histoire des asiles du Québec de la même époque : le sous-financement gouvernemental. André M. Paradis, «Le sous-financement gouvernemental et son impact sur le développement des asiles francophones au Québec (1845-1918) », RHAF, 50,4 (printemps 1997) : 571598 et « L'asile québécois et les obstacles à la médicalisation de la folie (1845-1890) », Bulletin canadien d'histoire de la médecine/Canadian Bulletin of Medical History, 11,2 (1994) : 297-334.

10. Voir notamment D. J. Rothman, The Discovery of the Asylum (Boston, Little, Brown and Company, 1971), 376 p.; A. T. Scull, Museums of Madness (Harmondsworth, Penguin Books, 1979), 275 p.; R. Castel, L'ordre psychiatrique (Paris, Les Éditions de Minuit, 1976), 334 p. Au Québec, A. Cellard a étudié l'avènement de l'asile dans une perspective de contrôle social exercé par les élites bourgeoises; voir A. Cellard, op. cit., 199. Pour Rothman, la diffusion du modèle asilaire aux ÉtatsUnis, durant la première moitié du XIX ${ }^{\mathrm{e}}$ siècle, constitue une réponse logique à la perception, par les Américains d'alors, d'un ordre social menacé. D'après Scull, c'est l'économie capitaliste qui expliquerait la mise en place d'un réseau d'asiles en Angleterre au XIX ${ }^{\mathrm{e}}$ siècle. Pour la période antérieure, la thèse classique (mais discutée) reste celle de Michel Foucault et de son Histoire de la folie à l'âge classique (Paris, Gallimard, 1972), 583 p. Pour ce dernier, le grand mouvement d'internement du $\mathrm{XVII}{ }^{\mathrm{e}}$ siècle, qui a frappé les fous comme d'autres déviants, aurait été déterminé par un changement dans l'éthique de l'époque, ceux qui se trouvèrent enfermés ayant contrevenu à la raison, à la nouvelle sensibilité face à la pauvreté, à l'inactivité, au travail et à l'ordre moral. 
sation et l'urbanisation n'engendrent pas une intolérance plus grande à la folie; où les réponses mises en œuvre au niveau local (mesures ad hoc, recours à l'appareil judiciaire, envoi éventuel à l'asile) ne représentent pas des instruments de contrôle des masses pauvres, mais bien des remèdes venant soulager des situations familiales problématiques. Dit autrement, il n'y a pas que l'asile qui importe aux XIX ${ }^{\mathrm{e}}$ et $\mathrm{XX}^{\mathrm{e}}$ siècles. Laissons de côté cette institution pour observer d'autres mécanismes sociaux.

\section{ETTRE INTERDIT POUR TROUBLES MENTAUX}

C'est à André Cellard que revient le mérite de la mise en valeur des dossiers d'interdiction et de curatelle ${ }^{11}$. Relevant du droit civil, ces documents représentent les traces des procédures légales par lesquelles un individu se voyait interdit, soit officiellement privé de la disposition de sa personne et de la gestion de ses biens ${ }^{12}$, et placé sous la gouverne d'un curateur, d'un tiers qui allait administrer sa personne et ses biens une fois l'interdiction prononcée.

Qui vise-t-on, au tournant du siècle, par l'interdiction pour folie? L'article 325 du Code civil, en vigueur lors de cette période, précise : «Le majeur ou le mineur émancipé qui est dans un état habituel d'imbécillité, démence ou fureur, doit être interdit, même lorsque cet état présente des intervalles lucides ${ }^{13}$. » Notons que sont aussi passibles d'interdiction les prodigues, les ivrognes d'habitude (loi de 1870) et les individus faisant usage d'opium, de morphine ou d'un autre narcotique (loi de 1895) ${ }^{14}$. L'interdiction pour folie entraîne la nullité des actes faits ultérieurement par l'individu ${ }^{15}$. Comme dans le cas des mineurs émancipés et des enfants conçus mais non encore nés, les interdits reçoivent un curateur ${ }^{16}$. Ce curateur joue le même rôle qu'un tuteur ${ }^{17}$. Il « prend soin » de l'individu interdit, « le représente dans tous les actes civils [et] administre ses biens en bon père de famille ${ }^{18}[\ldots] »$.

11. Voir A. Cellard «La curatelle et l'histoire des maladies mentales au Québec », Histoire sociale/ Social History, 19,38 (novembre 1986) : 443-450. D'autres historiens, hors Québec, ont fait appel à des documents s'apparentant aux curatelles. Voir par exemple J. E. Moran, « Asylum in the Community : Managing the Insane in Antebellum America », History of Psychiatry, 9,34 (juin 1998) : 217-240.

12. L.-P. Sirois, Tutelles et curatelles (Québec, Imprimerie de L'Action Sociale, 1911), 388.

13. Université McGill, Centre de droit privé et comparé, Code civil, 1866-1980 (Montréal, Société québécoise d'information juridique, 1981), 136. Édition historique et critique établie par P.-A. Crépeau et J. E. C. Brierley.

14. L.-P. Sirois, op. cit., 393.

15. Université McGill, Centre de droit privé et comparé, op. cit., article 334, 138.

16. Ibid., article $338,146$.

17. Ibid., article $343,147$.

18. L.-P. Sirois, op. cit., 442. 
La procédure suit un déroulement précis. Un parent, un allié ou l'époux ${ }^{19}$ amorce le processus en produisant une requête sous serment acheminée soit à la Cour supérieure, à l'un de ses juges ou à son protonotaire, soit à la cour de circuit ou à l'un de ses juges ${ }^{20}$. Cette requête «doit contenir l'articulation des faits d'imbécillité, démence [ou] fureur $^{21}[\ldots] »$. Aspect d'une importance capitale, dans ces suppliques sont en principe exposés les comportements du supposé malade qui, aux yeux du requérant, relèvent de la folie. Des témoignages accompagnent parfois la requête.

Suit un interrogatoire du défendeur, mené soit par le juge, soit par le protonotaire. En théorie, le greffier le consigne par écrit, questions et réponses comprises ${ }^{22}$. Les sujets traités sont au choix du magistrat ${ }^{23}$. Le conseil de famille, appelé par un avis de convocation, donne par la suite son opinion sur la condition de l'individu menacé d'interdiction ${ }^{24}$. Sept parents et alliés les plus proches de l'intimé, à l'exclusion du requérant, le composent en priorité ${ }^{25}$. Au moment de rendre sa décision, le juge n'est pas obligé de suivre l'avis du conseil : il a « une grande discrétion ${ }^{26} »$. Il peut prononcer l'interdiction, rejeter la requête ou, solution mitoyenne, nommer un conseil judiciaire au défendeur ${ }^{27}$. On adjoint un tel conseil en principe «à celui qui, sans être complètement insensé [...], est cependant faible d'esprit ${ }^{28}[\ldots] »$, ce qui habituellement l'empêche dès lors de poser seul plusieurs gestes à caractère économique ${ }^{29}$. La nomination d'un curateur figure dans les sentences décrétant une interdiction $^{30}$. Ce curateur est choisi par le conseil de famille et cette charge, officiellement conférée par le magistrat, peut revenir au

19. Université McGill, Centre de droit privé et comparé, op. cit., article 327, 136.

20. L.-P. Sirois, op. cit., 398.

21. Université McGill, Centre de droit privé et comparé, op. cit., article 328, 137.

22. L.-P. Sirois, op. cit., 402.

23. Ibid.

24. Université McGill, Centre de droit privé et comparé, op. cit., article 329, 137.

25. L.-P. Sirois, op. cit., 27 et 400.

26. Ibid., 404.

27. Université McGill, Centre de droit privé et comparé, op. cit., article 331, 138. Voir également L.-P. Sirois, op. cit., 404.

28. Université McGill, Centre de droit privé et comparé, op. cit., article 349, 149.

29. L.-P. Sirois, op. cit., 485.

30. Université McGill, Centre de droit privé et comparé, op. cit., article 341, 147. 
requérant ${ }^{31}$. Dernier détail, un certificat médical figure dans les dossiers d'individus déjà internés ${ }^{32}$.

Le présent travail s'appuie sur l'analyse de 138 documents de ce type ${ }^{33}$. Bien que limité, ce corpus met en lumière les caractéristiques sociologiques d'une population particulière de malades mentaux, les infractions aux normes qui leur sont reprochées et le rôle joué par les familles et la communauté dans leur prise en charge et exclusion. Il permet aussi de connaître les modalités de la présence médicale lors de ces procès, ainsi que la fréquence du recours à l' asile ${ }^{34}$. Évidemment, toutes les personnes qui souffraient aux yeux de leurs proches de désordres de l'esprit n'étaient pas interdites. Mais notre analyse laisse entrevoir certaines réalités laissées dans l'ombre par une histoire qui s'est surtout préoccupée de l'asile.

\section{DES HOMMES D'ORIGINE BRITANNIQUE HABITANT LES CAMPAGNES}

Les malades mentaux du district judiciaire de Saint-François qui ont fait face à la justice civile entre 1880 et 1920 montrent des caractéristiques sociologiques particulières. L'examen des variables du sexe, de l'âge, de l'état civil, du groupe linguistique, de l'habitat et de l'occupation montre que la folie traduite en justice offre un tout autre visage que

31. L.-P. Sirois, op. cit., 433 et 436.

32. Fait primordial, une seule modification est apportée aux articles du Code civil liés à l'interdiction et à la curatelle des malades mentaux entre 1880 et 1920. À partir de 1900, on n'interroge plus les personnes déjà enfermées dans un asile. En revanche, «il est produit un certificat du surintendant médical de cet asile constatant l'état mental du défendeur ». Voir Université McGill, Centre de droit privé et comparé, op. cit., article 330a, 137. C'est là un signe évident de l'accroissement du pouvoir d'un spécialiste médical, l'aliéniste. Cette constance de la procédure nous garantit une série documentaire dont les différentes pièces, les dossiers, ont obéi aux mêmes règles de production. Les années 1880 connaissent aussi, il est vrai, une grande effervescence législative en ce qui a trait aux asiles. Cependant, la seule disposition législative en mesure de nous intéresser est, semble-t-il, la loi de 1884 sur les asiles d'aliénés. Elle stipule qu'un administrateur provisoire, jouant le même rôle qu'un curateur, peut être nommé au patrimoine de gens internés dans un asile mais non interdits. Voir Acte relatif aux asiles d'aliénés subventionnés par la province de Québec, Statuts du Québec 1884, 47 V., c. 20, art. 45. P. Keating indique que, comme cet administrateur provisoire joue le même rôle qu' un curateur, l'internement et l'interdiction se trouvent alors placés sur un pied d'égalité. La procédure civile perdrait de ce fait un peu de son importance aux mains de l'asile dans les voies empruntées par la société québécoise pour régler le sort de ses fous. Voir P. Keating, op. cit., 95-96.

33. Parmi ces 138 documents, mentionnons la présence de 15 nominations de conseil judiciaire pour faiblesse d'esprit, de 2 requêtes en interdiction pour folie rejetées et de 6 procédures d'interdiction pour folie interrompues. Une dizaine de dossiers d'interdiction sont absents du fonds pour la période considérée. La série documentaire est donc presque exhaustive.

34. Faute de place, cet article ne s'attarde pas aux normes juridiques de définition de la folie qui peuvent être approchées par l'examen systématique des questions posées lors des interrogatoires. Pour une telle démarche, voir G. Boisclair, op. cit. 
celui des masses pauvres qui échouent à l'asile ${ }^{35}$. Quantité de facteurs viennent régir sa sanction judiciaire.

Certaines données, tout d'abord, trahissent l'importance de la mainmise sur le patrimoine familial dans le recours aux magistrats. Parmi les 138 causes, $98(71 \%)$ concernent des hommes et $40(29 \%)$ des femmes. Cette disproportion reflète bien la totale inégalité juridique et économique existant entre l'un et l'autre sexe à l'époque considérée. Les femmes mariées sont alors «frappées d'incapacité légale dans presque toutes les sphères de l'activité publique ${ }^{36}[\ldots] »$, se retrouvant de fait rangées parmi les incapables, au même titre que les interdits ${ }^{37}$. Il importe moins, dès lors, d'interdire une épouse dont le mari vit encore pour lui enlever des droits qu'elle ne possède pas. Toutefois, comme le fait remarquer L.-P. Sirois, la femme mariée peut être interdite ${ }^{38}$. Mais sa dépendance légale doit certainement contribuer à la faible représentation de la gent féminine dans notre population.

Nous ne connaissons l'âge que dans 77 cas. Il est révélateur que l'âge moyen s'établisse à 54 ans. Les procédures civiles destinées aux malades mentaux affectent peut-être la plupart du temps des gens d'âge mûr, et même des personnes assez âgées pour cette période. On appellerait la justice à la rescousse lorsque les facultés intellectuelles de l'individu, diminuées ou anéanties, ne lui permettent plus de gérer avec autant d'efficacité le patrimoine familial ou la part de celui-ci qui lui revient. Chez les 55 hommes dont l'âge est mentionné, la moyenne s'établit à 51 ans, contre 62 ans pour les 22 femmes dont le dossier contient la même donnée.

Regrouper les données du sexe, de l'âge et de l'état civil permet d'approfondir l'analyse. Le tableau 1 montre le nombre et l'âge moyen des personnes de l'un et l'autre sexe mariées, célibataires, veuves, séparées ou dont l'état civil est inconnu. L'importance des sous-groupes suivants retient l'attention : celui des 41 hommes mariés assez âgés (59 ans en moyenne) et plus encore celui des 19 veuves en fin de parcours (70 ans en moyenne). La réponse judiciaire civile à la folie affecterait donc plus souvent qu'à leur tour ces deux sous-ensembles. L'homme d'un certain âge, chef juridique et leader économique de l'unité de production familiale, doit subir en ces qualités une plus grande «surveillance » de ses proches qui, évidemment, dépendent de sa bonne conduite de la ferme ou du commerce. Pour la

35. Voir D. J. Rothman, op. cit., 283-284, ainsi que A. T. Scull, op. cit., 240 et ss.

36. Bettina Bradbury, Familles ouvrières à Montréal. Âge, genre et survie quotidienne pendant la phase d'industrialisation (Montréal, Boréal, 1995), 59.

37. Paul-André Linteau, René Durocher et Jean-Claude Robert, Histoire du Québec contemporain, 1 : De la Confédération à la crise (1867-1929) (Montréal, Boréal Express, 1979), 222.

38. L.-P. Sirois, op. cit., 390. 
femme, l'état plus autonome de célibataire ou de veuve appelle de façon plus ardente l'intervention de l'entourage. Ces deux situations, célibat ou veuvage, sont vécues par 28 des 40 individus de sexe féminin du groupe.

TABLEAU 1

SEXE, ÂGE* ET ÉTAT CIVIL

\begin{tabular}{lrccc} 
État civil & \multicolumn{2}{c}{ Hommes } & \multicolumn{2}{c}{ Femmes } \\
& Nombre & Âge moyen & Nombre & Âge moyen \\
Marié(e)s & 41 & 59 & 7 & 70 \\
Célibataires & 16 & 41 & 9 & 53 \\
Veufs et veuves & 3 & 65 & 19 & 70 \\
Séparé(e)s & 1 & 44 & 1 & inconnu \\
Inconnus & 37 & 45 & 4 & 40 \\
Total & 98 & - & 40 & -
\end{tabular}

Source : dossiers d'interdiction, Saint-François, 1880-1920.

Comme l'âge n'est disponible approximativement qu'une fois sur deux, voici le nombre de cas ayant servi au calcul de chacune des moyennes d'âge : 22 hommes et 2 femmes mariés, 12 hommes et 5 femmes célibataires, 3 veufs et 12 veuves, 1 homme séparé, 17 hommes et 3 femmes dont l'état civil est inconnu.

La répartition des interdits selon la langue pose, quant à elle, une série de questions tant démographiques et socio-économiques que culturelles. En effet, de 1880 à 1920, 95 anglophones (68,8\% du corpus) et 43 francophones (31,2\% du corpus) du district passent du côté des incapables ${ }^{39}$. La répartition linguistique des interdits, avant et à partir de 1901, est révélée par le tableau 2. Le problème est de taille. Les anglophones font de toute évidence bien plus appel que les francophones à la justice civile pour gérer la présence parmi eux d'aliénés, puisque, entre 1880 et 1920 , la population du district judiciaire devient très rapidement à forte majorité francophone $\mathrm{e}^{40}$. La proportion de francophones y passe de $45,4 \%$ en 1881 à $60,7 \%$ en 1901 et à $73,5 \%$ en 1921 , alors que

39. L'appartenance à l'un ou l'autre des groupes linguistiques a été établie d'après les patronymes.

40. Au sujet de l'évolution de la présence des francophones dans les Eastern Townships, majoritaires dans cette région dès 1881, on consultera Ronald Rudin, «The Transformation of the Eastern Townships of Richard William Heneker, 1855-1902 », Journal of Canadian Studies/Revue d'études canadiennes, 19,3 (automne 1984) : 39, et, du même auteur, " Naissance et déclin d'une élite locale : la banque des Cantons de l'Est, 1859-1912», RHAF, 38,2 (automne 1984) : 174. 
la part d'anglophones dégringole de $51,6 \%$ en 1881 à $38,0 \%$ en 1901 , pour finalement ne représenter qu'un mince $25,3 \%$ de la population de l'endroit en $1921^{41}$.

TABLEAU 2

\section{RÉPARTITION DES DOSSIERS SELON LA LANGUE}

\begin{tabular}{lccccr} 
Période & \multicolumn{2}{c}{ Anglophones } & \multicolumn{2}{c}{ Francophones } & Total \\
& Nombre & $\%$ & Nombre & $\%$ & \\
$1880-1900$ & 39 & 78,0 & 11 & 22,0 & 50 \\
$1901-1920$ & 56 & 63,6 & 32 & 36,4 & 88 \\
Total & 95 & - & 43 & - & 138
\end{tabular}

Source : dossiers d'interdiction, Saint-François, 1880-1920.

Peut-on penser que le domaine public (donc des institutions comme la justice et l'asile) occupe vraiment une plus grande place dans le fonctionnement des communautés anglo-saxonnes, alors que le privé, la famille et ses réseaux demeureraient plus essentiels pour les Canadiens français? Le constat de l'utilisation proportionnellement beaucoup plus élevée de la justice civile par les premières dans la régulation de la déviance mentale incite à poser la question. Pour y répondre, il faudrait pouvoir mesurer, pour les interdits des deux groupes, l'importance et l'étendue des réseaux de parentèle et d'alliance : une plus grande proximité de parents et d'alliés pourrait favoriser la prise en charge non officielle de l'incapable. À l'inverse, l'éloignement ou la faiblesse du réseau de parents pousseraient à faire appel à l'appareil judiciaire. Selon certains chercheurs, les Britanniques, industrialisés précocement, posséderaient une « culture » du recours à l' asile, institution apparue plus tôt chez eux ${ }^{42}$. Ces éléments pourraient expliquer la promptitude des anglophones à disposer, à l'aide de moyens exogènes à la communauté, d'un individu dérangeant. Malheureusement, dans l'état actuel de la recherche, toute conclusion à ce sujet demeure très prématurée.

Cependant, le facteur démographique mérite quand même d'être considéré. Comme on l'a vu, la moyenne d'âge des interdits est assez élevée. Or la population d'origine britannique des cantons est

41. Recensements du Canada de 1881, 1901 et 1921.

42. C'est ce qu'affirment A. Cellard et D. Nadon, « Ordre et désordre : le Montreal Lunatic Asylum et la naissance de l'asile au Québec », RHAF, 39,3 (hiver 1986) : 364-365. 
vieillissante, en raison d'une baisse plus rapide de sa natalité et de l'exode de ses jeunes ${ }^{43}$. Cet élément pourrait contribuer à la disproportion présente entre les deux groupes linguistiques parmi les interdits pour folie.

Le niveau de fortune des intimés joue sans doute aussi un rôle : L.-P. Sirois, juriste, concède que « l'interdiction n'est généralement prononcée que contre celui qui a des biens à sauvegarder ${ }^{44}{ }$. Les Britanniques, établis les premiers sur les meilleures terres et tenant le haut du pavé de l'économie de la région ${ }^{45}$, sont probablement plus prospères, en moyenne, que les francophones dont plusieurs sont ouvriers à Sherbrooke ou fermiers peinant sur les terres ingrates de Richmond ou Wolfe. Les pertes dues à l'aliénation étant potentiellement plus élevées pour le groupe le mieux nanti, l'interdiction serait plus souvent invoquée chez eux contre un chef de famille unsound of mind. Cette procédure requiert évidemment des frais.

Au chapitre de l'habitat, précisons que seuls les résidants de Sherbrooke ont été considérés comme urbains dans cette étude ${ }^{46}$. Presque tous les interdits, soit 114 (82,6\% des cas), proviennent de zones rurales, contre seulement 18 de Sherbrooke $(13 \% \text { des cas })^{47}$. Cette cité rassemble, en 1921, 17,4\% des résidants du district (23 515 sur 135 104). L'image de campagnes plus solidaires, plus tolérantes à l'endroit de parents imbéciles ou en démence ne tient donc pas en ce qui a trait à l'interdiction. En outre, le clivage ville/campagne ne se superpose pas au partage linguistique, comme l'atteste le tableau 3. Les 81 anglophones de milieu rural forment le noyau dur d'interdits. L'industrialisation et l'urbanisation ne représentent pas, de toute évi-

43. Voir les intéressantes comparaisons faites par J. I. Little entre Écossais et Canadiens français du canton de Winslow dans son ouvrage Crofters and Habitants. Settler Society, Economy, and Culture in a Quebec Township, 1848-1881 (Montreal, McGill-Queen's University Press, 1991), 81 et 92-98. Cette piste d'explication nous a été suggérée par Peter Gossage. L'exode des jeunes générations réduit d'autant le nombre de personnes disponibles pour s'occuper de parents séniles, ce qui rejoint notre hypothèse concernant les réseaux de parentèle.

44. L.-P. Sirois, op. cit., 409.

45. Pour s'en convaincre, il suffit de consulter le recueil de biographies de notables de V. E. Morrill et E. G. Pierce, Men of Today in the Eastern Townships 1917 (Sherbrooke, Sherbrooke Record Company, 1916), 297 p.

46. Entre 1880 et 1920 , dans le district judiciaire de Saint-François, on ne voit pas quelle autre agglomération pourrait prétendre au titre de ville en considérant les implications énormes d'un mode de vie vraiment urbain au point de vue de la sociabilité et des mentalités. Hormis Sherbrooke, seule la petite ville de Magog présente au cours de cette quarantaine d'années une population approchant les 5000 habitants (5159 en 1921). Voir Sixième recensement du Canada, 1921, I : Population (Ottawa, F. A. Acland, 1924), 235-236 et 242.

47. Dans six cas, le lieu de résidence est inconnu. 
TABLEAU 3

\section{GROUPES LINGUISTIQUES ET HABITAT}

\begin{tabular}{lccccr} 
Habitat & \multicolumn{2}{c}{ Anglophones } & \multicolumn{2}{c}{ Francophones } & Total \\
& Nombre & $\%$ & Nombre & $\%$ & \\
Campagne & 81 & 90,0 & 33 & 78,6 & 114 \\
Ville & 9 & 10,0 & 9 & 21,4 & 18 \\
Total $^{*}$ & 90 & 100,0 & 42 & 100,0 & 132
\end{tabular}

Source : dossiers d'interdiction, Saint-François, 1880-1920.

* L'habitat de cinq anglophones et d'un francophone est inconnu.

dence, les conditions majeures d'une plus grande intolérance à la folie en ce qui concerne l'interdiction ${ }^{48}$.

Enfin, la thèse de la domination de classe s'avère à son tour impropre pour appréhender la gestion des troubles mentaux dans le cas présent, comme le montre l'examen des occupations des intimés. Seuls 87 dossiers recèlent des informations à ce sujet ${ }^{49}$. Toutes les professions relevées furent réparties dans les catégories professionnelles suivantes : ouvriers, ouvriers spécialisés, farmers et cultivateurs, cols blancs, marchands et commerçants, professions libérales, divers et cas imprécis (tableau 4). C'est la présence massive d'agriculteurs qui d'emblée s'impose, fait cependant peu étonnant pour une zone très rurale et pourvue d'une seule ville de taille moyenne, Sherbrooke. Malgré cela, les procédures judiciaires civiles destinées aux malades mentaux ne peuvent apparaître comme des instruments au service de l'élite; l'interdiction concerne toutes les classes sociales.

Les interdits de Saint-François présentent en somme des caractéristiques assez spécifiques. Dans ce groupe à la moyenne d'âge plutôt élevée, les hommes figurent en surnombre et, parmi eux, ressort le sous-groupe des hommes mariés, tandis que les femmes, moins nombreuses, sont la plupart

48. Au Québec, la thèse de l'influence de l'urbanisation dans la mise au pas asilaire de la folie a été soutenue par A. Cellard, op. cit., 209, et A. Cellard et D. Nadon, loc. cit., 365, et par A. BertrandFerretti, «Pratiques sociales et pratiques discursives : le discours sur la folie au Québec, sous l'Union », dans A. Paradis, dir., et al., op. cit., 107 et 147.

49. Les femmes vivant sous le toit paternel, les épouses et les veuves ont été munies respectivement de l'occupation de leur père, époux ou mari défunt. Ce n'est pas tant le métier exercé réellement par chacun des individus qui mérite attention, mais bien la position sociale attachée à l'exercice d'une profession soit par l'individu lui-même, soit par le chef de famille. 


\section{TABLEAU 4}

\section{LES OCCUPATIONS}

Catégories et exemples

Occurrences

6 7

45

4

8

farmer, turnkey rentier?, sailor?

Total

Source : dossiers d'interdiction, Saint-François, 1880-1920.

du temps veuves, célibataires et probablement d'âge avancé. C'est par contre la représentation remarquablement élevée des anglophones, presque tous campagnards, par rapport à leur poids réel dans la population du district, qui surprend le plus. Certains facteurs influencent donc la sanction judiciaire de la folie. C'est le cas de la possibilité de gérer le patrimoine familial, possibilité qui varie selon le sexe et l'état civil; l'appartenance linguistique soulève aussi toute une série d'interrogations. Le fait de résider en ville et la classe sociale paraissent toutefois ne pas avoir d'effet.

\section{LES INFRACTIONS AUX NORMES}

Quels sont, au tournant du siècle, les présupposés sous-jacents à l'établissement d'une barrière entre l'individu normal et l'aliéné? Bien que la variété des conduites dénoncées dans les dossiers relève un peu du capharnaüm, il est possible d'établir une typologie des comportements déviants. Quelques procès peuvent en outre révéler certaines particularités de la perception populaire de la maladie mentale à cette époque ${ }^{50}$. Disons-le

50. Nous avons délibérément insisté sur les situations montrant la spécificité de la perception de la folie au tournant du siècle. Dans cette section, seuls les témoignages de gens ordinaires furent retenus, à l'exclusion des interventions de magistrats ou de médecins. 
d'emblée, c'est de façon extrêmement nuancée qu'il faut aborder les préoccupations normatives ayant conduit à étiqueter un individu comme aliéné mental. Toute explication monolithique de la nouvelle place donnée au fou au XIX ${ }^{\mathrm{e}}$ siècle, tel un désir de la « bourgeoisie » de réguler les comportements populaires, paraît insatisfaisante à la suite de cet exercice.

Six formes de comportements ont été décrites à plus de 30 reprises dans des dossiers distincts. On peut voir là des signes communs d'aliénation pour la période 1880-1920. En ordre décroissant, ce sont : les troubles de la personnalité et les délires (53 occurrences), les cas d'atteintes à une saine gestion du patrimoine (47), le défaut clairement énoncé d'aptitudes intellectuelles ou physiques (45), les problèmes de communication verbale (42), la violence (38) et les entorses à la tranquillité publique (31). Il a toutefois paru préférable lors de l'analyse de répartir toutes les catégories à l'intérieur de trois grandes rubriques concernant successivement l'économie de la maisonnée, les situations de communication en général et les rapports aux bonnes mœurs.

Les dérèglements liés à la bonne gestion du patrimoine sont très fréquemment relevés ( 47 occurrences). On peut croire que cette préoccupation, même sans être explicitement évoquée, fonde en dernière instance une majorité de recours en justice pour interdire. On a tout avantage à ce que la ferme familiale ou la boutique ne sombrent pas dans l'abandon ou sous les mauvaises manœuvres. Ce qui ne réduit en rien, précisons-le, la variété des critères de définition de la folie que donnent à voir les requêtes et témoignages : si l'objectif ultime est économique, absolument rien n'empêche les requérants et témoins d'apporter des exemples ou des preuves n'ayant aucun caractère monétaire ou patrimonial (délire religieux, violence, etc.) qui établissent la folie et de là l'incapacité à voir à ses affaires. Plusieurs individus paraissent vraiment incapables d'accomplir des actes à caractère économique, telles les personnes déficientes depuis leur plus jeune âge. Mais, en cette matière, le regard sur la santé mentale de l'intimé est parfois assez subjectif. Ainsi, lors d'un procès, on demande à un témoin :

Ques. Voulez-vous donner quelques instances et circonstances dans sa conduite qui vous portent à croire qu'il est fou?

Rep. Je considère que sa conduite depuis à peu près six mois est une ruine complète pour lui-même. Je suis son voisin. Je crois qu'il s'est fait dommage pour un mille piastres le moins $[\text { sic }]^{51}$.

51. Cour supérieure, Sherbrooke (dorénavant CSS), fonds testament, boîte cs $4, \mathrm{n}^{\circ} 320$, 12 juillet 1882, nomination d'un conseiller judiciaire à Eli E. 
On dénonce également les manquements aux obligations professionnelles; ratés administratifs et dettes accumulées sont signalés. Une requête mentionne qu'une dame possède une propriété hypothéquée à $7 \%$ d'intérêt, alors que son compte en banque, qui n'a qu' un rendement de $3 \%$, est suffisant pour éliminer cette même hypothèque... ${ }^{52}$ Rappelons que la requête «doit contenir l'articulation des faits d'imbécillité, démence [ou] fureur ${ }^{53}$ [...] ». Dans un autre dossier, une propriété agricole qui n'a pas connu de progrès depuis deux ans et le non-paiement des intérêts de l'hypothèque et des taxes s'ajoutent à une longue liste de conduites déviantes ${ }^{54}$. La marge de manœuvre dans la gestion du patrimoine paraît assez réduite.

Le manque d'aptitudes physiques et intellectuelles est aussi fréquemment indiqué dans les requêtes et témoignages (45 occurrences). Ces déficits sont à maintes reprises reliés à des questions pratiques d'administration. En témoigne la fréquence du critère de l'incapacité à connaître le bon emploi de l'argent. Mais un cheminement scolaire raté peut à son tour étayer la démonstration d'inaptitude ${ }^{55}$. François G., au surplus, « n'a pas pu faire sa première communion, c'est un imbécile ${ }^{56}[\ldots] »$.

En revanche, les accusations explicites d'improductivité ou de fainéantise n'apparaissent que rarement (9 occurrences). Certaines situations se révèlent quand même significatives. Le rôle à jouer au foyer, à l'époque, varie évidemment selon le sexe, et l'anormalité-folie de la femme diffère à ce titre de celle de l'homme. Un fermier du canton de Compton se plaint de sa sœur qui réside avec lui : elle ne peut faire qu'un peu de travaux ménagers sous la direction de quelqu'un, a une faible santé et n'est capable ni de coudre ni de cuisiner ${ }^{57}$. C'est l'antithèse de son genre. Cette prestation tout à fait insuffisante participe à la démonstration de son aliénation mentale.

On établit aussi des rapports très formels entre prodigalité et troubles mentaux ( 21 occurrences). Ici encore, la marge de manœuvre en affaires paraît assez restreinte, et la surveillance de la communauté, parfois étroite. Un commerçant, faisant référence à la maladie de son propre fils, témoigne : «Je considère que sous les circonstances ou [sic] Mons. [E.] m'a offert de l'argent pour envoyer aux sœurs pour faire dire des prières était un signe d'insanité — parce qu'il n'a jamais rien donné dans ce but

52. CSS, fonds tutelle curatelle (dorénavant FTC), $\mathrm{n}^{\circ} 112,10$ mai 1886, interdiction et curatelle d'Ann W.

53. Université McGill, op. cit., article 328, 137.

54. CSS, FTC, $\mathrm{n}^{\circ} 2582,11$ octobre 1909, interdiction et curatelle de Frederick T.

55. CSS, FTC, $\mathrm{n}^{\circ}$ 1286, 25 novembre 1898, interdiction et curatelle d'Edward C.

56. CSS, FTC, $\mathrm{n}^{\circ} 3353,21$ octobre 1913, interdiction et curatelle de François G.

57. CSS, FTC, $\mathrm{n}^{\circ}$ 999, 16 mai 1896, interdiction et curatelle de Sarah M. 
là [sic] [à] ma connaissance et parce qu'il était trop ménager et trop avare pour cela ${ }^{58}$. » Requérants et témoins ne s'en tiennent pas, loin s'en faut, à ne relever que des actions de mauvaise gérance.

Ainsi certaines situations problématiques peuvent être regroupées autour de la question des liens à entretenir avec l'entourage et la communauté. C'est là un deuxième grand « secteur » de la déviance. Les problèmes de communication, notamment, constituent un des symptômes les plus souvent mentionnés ( 42 occurrences). On trouve ici absolument de tout : perte des facultés de bien comprendre, de s'exprimer avec aisance, d'être lucide, etc. De toute évidence, il y a des cas graves :

[...] le dit [É]mile [D.] depuis le quatrième jour de février courant est tombé dans un état de démence et d'aliénation mentale [et] le dit [É]mile [D.] depuis qu'il est dans cet état parle des heures de temps en articulant des mots qui n'ont pas de suite et pas de sens [...] ces mots qu'il articule ainsi ne signifient rien $[\ldots]^{59}$.

L'errance représente pour sa part un indice moins commun d'anormalité ( 20 occurrences). Quitter sa résidence sans but précis peut révéler le fou ou la folle. Les premiers actes d'imbécillité et d'insanité de Sarah M. furent, dit-on, ses escapades du domicile paternel dans le canton de Compton, parfois la nuit ${ }^{60}$. Un chef de famille menace de ramasser ses avoirs et d'abandonner les siens ${ }^{61}$ : il va sans dire qu'un tel projet se trouve d'office signalé au magistrat en exercice.

Les troubles de la personnalité, c'est-à-dire les traits de caractère exagérés altérant le fonctionnement de l'individu, associés aux délires, occupent le premier rang des conduites dénoncées (53 occurrences). Ces deux types d'affections constituent conjointement la manifestation la plus courante et la plus évidente d'insanity aux yeux des habitants des Cantons de l'Est. Des traits caractériels exacerbés s'avèrent probablement gênants à l'intérieur de petites communautés rurales. Narcissisme et délire des grandeurs affectent quelques personnes. Mais, même dans ces cas-là, certaines situations poussent le chercheur à s'interroger sur la force de la norme sociale à cette époque. Un témoin dit de l'un de ces individus qu'il l'a « [...] toujours pris pour un homme qui a la tête craquée [sic]. Depuis le mois d'avril dernier il n'a plus voulu écouter les conseils de sa femme et

58. CSS, fonds testament, boîte cs $4, n^{\circ} 320,12$ juillet 1882 , nomination d'un conseiller judiciaire à Eli E.

59. CSS, FTC, $\mathrm{n}^{\circ}$ 801, 22 février 1894, interdiction et curatelle d'Émile D.

60. CSS, FTC, no 999, 16 mai 1896, interdiction et curatelle de Sarah M.

61. CSS, FTC, $n^{\circ}$ 600, 13 novembre 1891, interdiction et curatelle de Thomas $\mathrm{S}$. 
veut être indépendant de tous ${ }^{62} \gg$. États certainement plus graves, les hallucinations et délires permettent cependant d'accéder à quelques points de repère du «paysage mental » du tournant du siècle. La parole du fou, débridée, laisse libre cours à l'expression de représentations probablement extériorisées d'une autre manière par les gens «normaux ». On peut relever quatre thèmes majeurs parmi ces divagations. D'abord, la richesse et la ruine. David M., fermier du canton de Dudswell, a offert d'acheter la Merchant's Bank ${ }^{63}$. Deuxièmement, la reine Victoria dut bien s'ancrer dans l'imaginaire collectif de ces contrées bien pourvues en habitants d'origine britannique. Ainsi, quand John A. tente d'incendier sa maison, sa femme essaie d'éteindre les flammes : il lui dit de n'en rien faire, que la reine allait s'occuper d'elle et de ses enfants... ${ }^{64}$ Point d'étonnement non plus qu'à une époque de forte pratique religieuse et de grande présence des églises dans la société, la religion soit un véhicule privilégié des différentes divagations et visions. Robert D., un mystique persuadé d'être un agent spécial de Dieu, se lève durant un religious meeting du canton de Barnston pour relater sa vision de l'étoile de Bethléem ${ }^{65}$. Enfin, dernier thème et souci de toutes les époques, la mort hante et obsède plusieurs malades en passe d'être interdits.

Troisième et dernier grand domaine de non-conformité : les entorses aux bonnes mœurs et à la morale. La violence du fou (38 occurrences), ses atteintes à la tranquillité publique (31) et ses bizarreries diverses (23) viennent appuyer maintes requêtes. Rares sont les transgressions spécifiques que sont l'irréligion (5), l'intempérance (3) et les inconduites sexuelles (13), mais leur présence dans les dossiers est éloquente.

Nombre d'individus apparaissent comme porteurs de violences éventuelles. Les risques n'ont souvent rien d'imaginaire. La femme de Napoléon T., requérante, raconte : « [...] il a cherché à m'étrangler plusieurs fois, et si on n'était pas venu à mon secours j' aurais été étouffée; il m'a frappé $[s i c]$ avec un manche de hache, avec une chaise, des fers a $\left[\right.$ sic] repasser et tout ce qu'il pouvait attraper ${ }^{66}[\ldots] »$. Par ailleurs, bien des intimés troublent la paix des familles et du voisinage par leur tapage et par des expéditions nocturnes. Un homme, par moments, crie, chante

62. CSS, fonds testament, boîte cs $4, \mathrm{n}^{\circ} 320,12$ juillet 1882 , nomination d'un conseiller judiciaire à Eli E.

63. CSS, FTC, $\mathrm{n}^{\circ} 278,18$ mars 1886 , interdiction et curatelle de David M.

64. CSS, FTC, no 394, 9 septembre 1889, interdiction et curatelle de John A.

65. CSS, FTC, $\mathrm{n}^{\circ} 229,28$ novembre 1887 , nomination d'un conseiller judiciaire à Robert $\mathrm{D}$.

66. CSS, FTC, $\mathrm{n}^{\circ} 626,8$ avril 1892, interdiction et curatelle de Napoléon T. 
et prie sur la route. Son frère le menace alors comme suit : « if I hear any more loud shouting or disturbance on the streets I will have to send you to Beauport. » Il fait bien sûr référence à l'asile qui s'y trouve ${ }^{67}$.

Bien qu'exceptionnellement rencontrée, la présence même de l'irréligion au sein de documents visant à prouver le dérangement d'individus est révélatrice. Eloïse B., " atteinte d'aliénation mentale depuis près d'une année [...] ne prie plus, bien qu'ayant été autrefois une personne très pieuse $\mathrm{e}^{68} \gg$. Edward C., pour sa part, trinque à l'excès dès qu'il a de l'argent ${ }^{69}$. Enfin, quelques cas d'indécence soulignent à leur manière la pudeur des usages du temps. On en parle aussi à mots couverts, peut-on croire : une telle « a fait des actes devant moi $^{70}[\ldots] »$, affirme un témoin. Il semble inadmissible que des personnes âgées un peu confuses paraissent le matin hors de leur chambre en robe de nuit ${ }^{71}$. Pire, Edith M. s'enfuit de chez elle très légèrement vêtue et se cache de ses parents ${ }^{72}$.

Requérants et témoins, tout en apportant ce qu'ils tiennent pour des preuves d'aliénation, parlent d'eux-mêmes, du tournant du siècle et de ses règles normatives. Quand il y a exclusion, le groupe dominant s'affirme, étale l'image qu'il voudrait voir régner partout ${ }^{73}$. Le repérage des critères populaires de définition de la maladie mentale permet de débuter à la « racine du mal », soit avec les regards de ceux et celles aux prises en première ligne avec les transgressions de normes. Toute histoire de la folie devrait commencer par là, et non avec les préoccupations des « élites ».

\section{LA FAMILLE ET L'ENTOURAGE INTERVENTION PREMIÈRE ET MESURES D'URGENCE}

On doit accorder un rôle prépondérant à ces regards, aux conceptions et représentations ayant cours dans la population et ce, malgré la grande place que paraissent occuper les officiers de justice dans le déroulement des affaires qui nous occupent. La procédure officielle d'interdiction canalise une des réactions de l'entourage face au déviant. Cet entourage a l'initiative, le choix d'y faire appel ou non : elle est à son service. Le système judiciaire reçoit les requêtes. L'article 325 du Code civil stipulant l'interdiction des malades mentaux ne donne pas lieu, à ce que l'on

67. CSS, FTC, $\mathrm{n}^{\circ} 229,28$ novembre 1887, nomination d'un conseiller judiciaire à Robert D.

68. CSS, FTC, $\mathrm{n}^{\circ}$ 3702, 28 juin 1916, interdiction et curatelle d'Éloïse B.

69. CSS, FTC, $\mathrm{n}^{\circ} 1286,25$ novembre 1898, interdiction et curatelle d'Edward C.

70. CSS, FTC, $\mathrm{n}^{\circ} 3702,28$ juin 1916, interdiction et curatelle d'Élö̈se B.

71. CSS, FTC, no 1994, 3 avril 1905, nomination d'un conseiller judiciaire à Ida M.

72. CSS, FTC, $n^{\circ} 3062,22$ août 1911, interdiction et curatelle d'Edith M.

73. J.-C. Schmitt, «L'histoire des marginaux », dans J. Le Goff, dir., La nouvelle histoire (Bruxelles, Éditions Complexe, 1988), 300. Deuxième édition. 
sache, à une chasse aux aliénés dirigée par les élites, élites que, pourtant, bien des ouvrages présentent comme les actrices essentielles de la mise au pas de l'aliénation mentale.

De plus, l'intervention des proches ne se limite pas, loin de là, à demander l'interdiction : la cohabitation avec une personne malade mentale ou supposée telle requiert souvent la mise en œuvre de stratégies spécifiques et, à l'occasion, l'adoption de mesures d'urgence. Le voisinage apparaît dans plusieurs affaires comme un partenaire actif de la gestion de la présence d'individus au comportement hors normes.

Il arrive que soit prévu à long terme l'entretien d'un incapable. Un père ayant fait donation de tous ses biens meubles et immeubles à l'un de ses fils, « [...] par le même acte il [charge] le dit Charles [G.] de loger, nourrir et vêtir avec lui le dit François [G.], son frère, sa vie durant [...] ». Ce dernier, « agé $[s i c]$ de trente deux $[s i c]$ ans est depuis sa naissance idiot et en demense $[\text { sic }]^{74} \gg$. Les cas d'hébergement de personnes dont l'interdiction est demandée sont par ailleurs nombreux. Après un séjour de six mois au Longue Pointe Lunatic Asylum, George M. a résidé avec sa sœur et deux de ses frères, «but is still insane $\&$ of infirm mind ${ }^{75} »$. Un malade ou une personne très âgée en démence nécessitent évidemment quelques soins. Le beau-frère d'Éloïse B., qui l'héberge chez lui, dit qu' « elle ne connaît plus les règles élémentaires de la propreté [...] il faut en prendre soin comme d'un jeune enfant ${ }^{76}{ }$.

Certains individus demandent une surveillance constante et le recours à des formes d'enfermement à domicile est quelquefois nécessaire : on ferme la maison à clé pour empêcher Jane B. de sortir la nuit ${ }^{77}$. Le frère d'Émile D. est allé à l'endroit où habite ce dernier « pour y passer la nuit vu qu'il avait appris que ledit Émile [D.] était devenu fou». Ce dernier a des « $\operatorname{gardiens}^{78} »$. James G., alité chez son beau-frère, était si dément et violent avant son départ pour l'asile « that he had to be watched and guarded constantly for fear he might take his own life or attempt to do violence on the members of this deponent's family ${ }^{79} »$. Parents, amis et habitants des environs doivent aussi, à l'occasion, se livrer à des activités de recherche et de sauvetage. Un jeune trader du canton d'Eaton est introuvable durant 48 heures au mois de décembre 1888 : «After search was made for him, he was found secreted under about two feet of straw in his barn chilled and numb and almost unconscious [...]. »

74. CSS, FTC, $\mathrm{n}^{\circ} 3353,21$ octobre 1913, interdiction et curatelle de François G.

75. CSS, FTC, $\mathrm{n}^{\circ} 326,17$ décembre 1888, interdiction et curatelle de George M.

76. CSS, FTC, $\mathrm{n}^{\circ} 3702,28$ juin 1916, interdiction et curatelle d'Eloïse B.

77. CSS, FTC, $\mathrm{n}^{\circ} 1223,18$ mai 1898, interdiction et curatelle de Jane B.

78. CSS, FTC, n ${ }^{\circ} 801,22$ février 1894, interdiction et curatelle d'Émile D.

79. CSS, FTC, $\mathrm{n}^{\circ} 772,24$ novembre 1893, nomination d'un judicial adviser à James G. 
Son oncle par alliance et un tiers le mettent au lit, les voisins arrivant par la suite $^{80}$. L'intervention du voisinage peut revêtir d'autres formes : des citoyens viennent, par exemple, trouver le père de Frederick $T$. pour se plaindre de l'exhibitionnisme de celui-ci. Un homme se voit même obligé d'intervenir lorsque, à une occasion, il s'avance nu vers une petite fille ${ }^{81}$.

L'entourage et la communauté s'interposent parfois pour assurer la protection du patrimoine de certains individus. Un homme âgé se présente à une succursale de la Canadian Bank of Commerce et demande le retrait de tout son avoir déposé à cet endroit, soit $3000 \$$, d'après le témoin racontant cet épisode. Mais « one of the clerk who know his mental condition secured his signature to a check for $\$ 50$, gave him the amount and he went away satisfied ${ }^{82} »$. Un hotel-keeper de Richmond fait une offre bien trop élevée pour une petite propriété à une vente aux enchères : «Friends who were present knowing his condition of mind, interferred for his protection against having the said property for which he had no use, knocked down to him $[\ldots]^{83}$.»

Parfois des voisins interviennent lorsque la sécurité de ceux qui cohabitent avec l'individu « dérangé » est mise en danger. En raison de la violence de John A., un homme, en tant que voisin et ami, dit-il, s'est senti concerné par la sécurité de sa femme et de sa famille. Il procède conséquemment aux démarches nécessaires, en compagnie d'une autre personne, pour le faire conduire en prison ${ }^{84}$. On envoie chercher un hôtelier de Sherbrooke pour qu'il protège sa mère et ses sœurs aux prises avec un chef de famille violent à l'extrême. L'hôtelier doit même les cacher chez lui à une occasion ${ }^{85}$.

Mesures d'entretien à long terme, hébergement, soins, surveillance, protection du patrimoine, interventions en cas de danger pour l'entourage : la requête en interdiction est postérieure à ces interventions ad hoc décrites dans les dossiers. Ces gestes soulignent, à n'en pas douter, le caractère essentiel de l'instance familiale et communautaire dans les réponses à la folie, et ce même après la constitution d'un solide réseau d'asiles au Québec.

Mais qui amorce le rejet juridique de la folie? L'article 327 du Code civil stipule que « toute personne est admise à provoquer l'interdiction de

80. CSS, FTC, $\mathrm{n}^{\circ} 332,16$ janvier 1889 , interdiction et curatelle de Jonathan S.

81. CSS, FTC, $\mathrm{n}^{\circ}$ 2582, 11 octobre 1909, interdiction et curatelle de Frederick T.

82. CSS, FTC, $\mathrm{n}^{\circ} 3273,10$ mars 1913 , interdiction et curatelle d'Erastus B.

83. CSS, FTC, $\mathrm{n}^{\circ} 600,13$ novembre 1891 , interdiction et curatelle de Thomas S.

84. CSS, FTC, $\mathrm{n}^{\circ}$ 394, 9 septembre 1889, interdiction et curatelle de John A.

85. CSS, FTC, $\mathrm{n}^{\circ} 626,8$ avril 1892, interdiction et curatelle de Napoléon T. 
son parent ou allié prodigue, furieux, imbécille [sic] ou en démence; il en est de même de l'un des époux à l'égard de l'autre ${ }^{86}$ ». Dans 96 cas (69,6\% des requêtes), ce sont des parents et alliés intimes de l'individu (époux, épouses, pères, mères, fils, filles, frères et sœurs) qui prennent l'initiative. Les autres parents et alliés se font bien plus discrets. Et une fois l'interdiction prononcée, quelles relations rencontre-t-on le plus fréquemment entre les curateurs nommés et ceux dont le trouble mental vient d'être consacré? Le juriste L.-P. Sirois croit « que toute personne majeure, mâle et non interdite et frappée d'aucune incapacité peut être nommée ${ }^{87}[\ldots] »$. L'article 342 précise quand même que « le mari, à moins de raisons jugées valables, doit être nommé curateur à sa femme interdite. La femme peut être curatrice à son mari ${ }^{88} »$. En général, la charge de curateur ou de conseiller judiciaire échoit à un membre de la famille, parent ou allié ( $71,7 \%$ des nominations), et bien souvent à quelqu' un de très proche (époux, épouse, père, mère, fils, frère ou même sœur : 49,7\% des nominations $)^{89}$. L'exclusion des fous et folles de la vie civile apparaît dans les faits éminemment telle que la loi la veut : une question familiale.

\section{«SOFTENING OF THE BRAIN TISSUE » LA PRÉSENCE MÉDICALE}

Il reste que les membres de la famille et de l'entourage ne sont pas les seuls joueurs dans les affaires d'interdiction. La présence médicale y est forte. Dans 107 dossiers sur 138, soit dans la plupart des cas (77,5\%), un ou des médecins (aliénistes compris) furent appelés à donner leur avis d'une façon quelconque. On pourrait donc conclure à une assez forte médicalisation de l'aliénation mentale dans la justice civile à l'époque qui nous intéresse, ce qui viendrait conforter, même sur le plan local, la thèse de l'appropriation médicale de la maladie mentale au XIX ${ }^{\mathrm{e}}$ siècle. Une analyse assez fine des interventions faites en dehors du cadre asilaire conduit par contre à nuancer cet argument essentiel de l'historiographie de la folie.

Il convient de distinguer d'abord, parmi cette masse d'avis médicaux, les certificats émis par des surintendants d'asiles ou leurs assistants, donc émis par des spécialistes de la question, des simples témoignages des médecins des villes et villages des Cantons de l'Est. Soixante-trois dossiers contiennent des certificats provenant d'asiles et 45 renferment des témoignages

86. Université McGill, Centre de droit privé et comparé, op. cit., article 327, 136.

87. L.-P. Sirois, op. cit., 435. Cette opinion a souffert quelques exceptions pratiques.

88. Université McGill, Centre de droit privé et comparé, op. cit., article 342, 147.

89. Il n'y a pas de nominations dans huit dossiers (actions interrompues et rejetées). On compte aussi de très rares nominations de joint curators et de joint judicial advisers. 
de médecins de la région. Quelques documents (8) montrent à la fois des certificats asilaires et des témoignages de non-spécialistes ${ }^{90}$. Mais, dans tous les cas, rien ne semble astreindre l'homme de science à poser un diagnostic précis. Par conséquent, l'examen des verdicts des aliénistes et des médecins non spécialistes ne possède qu'une valeur indicative (tableaux 5 et 6 ).

Cette absence d'obligation d'émettre un diagnostic formel se traduit malheureusement par l'absence de verdict clair dans de nombreux dossiers. On se borne souvent à dire que l'individu est incapable de prendre soin de sa personne et de ses biens, référence explicite à la finalité de l'interdiction. Quelques substantielles différences apparaissent néanmoins entre les diagnostics rendus par les aliénistes et ceux prononcés par les médecins des cantons. L'asile accueille probablement les cas les plus lourds. D'où, croyons-nous, le nombre important d'internés souffrant de démence sous toutes ses formes ( 23 cas, tableau 5), alors qu'aucun cas de ce genre (hormis 2 cas de sénilité, il est vrai) n'est relevé chez les aliénés jouissant encore de leur liberté. La démence est définie à l'époque comme une « affection cérébrale caractérisée par la perte plus ou moins complète des facultés intellectuelles, morales et affectives ${ }^{91} »$.

Parmi les malades demeurés dans leurs familles, on n'indique pas non plus de cas de mélancolie, état se signalant «par un état général de dépression ${ }^{92} »$, de paralysie générale, « affection chronique caractérisée par la déchéance du pouvoir musculaire et par de la démence ${ }^{93} »$, ou de folie des dégénérés ${ }^{94}$. Clientèles différentes ou nosographies dissemblables? Difficile à dire. Les aliénistes sont sans aucun doute plus au fait des dernières théories médicales sur la folie. Le constat le plus intéressant réside dans l'emploi, par les médecins des cantons, du diagnostic de softening of the brain, absent chez les aliénistes. Peut-être ceux-ci ont-ils abandonné cette entité nosologique encore présente en 1890 dans un sérieux traité de médecine en tant que maladie de l' « appareil nerveux ${ }^{95}$ ».

Plusieurs praticiens des cantons ont laissé des témoignages assez éloquents de leur examen clinique de malades. Leur expertise, dans un

90. Sept dossiers font référence à une intervention médicale sans contenir de certificats ou de témoignages.

91. Traité élémentaire de matière médicale et guide pratique des Sours de Charité de l'asile de la Providence (Montréal, Imprimerie de la Providence, 1890), 1342. Troisième édition revue, corrigée et augmentée de tous les remèdes nouveaux depuis 1870.

92. Ibid., 1339.

93. Ibid., 1341.

94. D'après le Traité élémentaire..., « les signes psychiques [de la dégénérescence] consistent dans l'absence ou l'insuffisance du développement des facultés intellectuelles et morales ». Voir Traité élémentaire..., op. cit., 1344. Cette référence nous a été aimablement fournie par Denis Goulet.

95. Ibid., 1124. 


\section{TABLEAU 5}

\section{DIAGNOSTICS DES CERTIFICATS MÉDICAUX ASILAIRES}

\begin{tabular}{|c|c|c|}
\hline \multicolumn{2}{|r|}{ Diagnostics et exemples } & Nombre \\
\hline Démence & $\begin{array}{l}\text { Démence, aliénation mentale et démence, aliénation et en état } \\
\text { de démence, état de démence complète, dementia, demented } \\
\text { condition } \\
\text { Epileptic dementia } \\
\text { Chronic dementia } \\
\text { Secondary dementia } \\
\text { Secondary or terminal dementia } \\
\text { Senile dementia }\end{array}$ & $\begin{array}{c}16 \\
1 \\
2 \\
1 \\
1 \\
2\end{array}$ \\
\hline Mélancolie & $\begin{array}{l}\text { Mélancolie } \\
\text { Mélancolie avec stupeur } \\
\text { Simple melancholia } \\
\text { Acute melancholia (attack of) }\end{array}$ & $\begin{array}{l}2 \\
1 \\
1 \\
1\end{array}$ \\
\hline Manie & $\begin{array}{l}\text { Manie } \\
\text { Hypermanie anxieuse (attack of) } \\
\text { Chronic hypermania (manie chronique selon le même } \\
\text { médecin) }\end{array}$ & $\begin{array}{l}2 \\
1 \\
1\end{array}$ \\
\hline $\begin{array}{l}\text { Paralysie } \\
\text { générale }\end{array}$ & $\begin{array}{l}\text { Paralysie générale progressive } \\
\text { General paralysis of the insane }\end{array}$ & $\begin{array}{l}1 \\
1\end{array}$ \\
\hline Autres & Folie des dégénérés & 1 \\
\hline Imprécis & $\begin{array}{l}\text { Aliénation mentale, aliéné, maladie mentale, maladie mentale } \\
\text { chronique, insane, insanity, mental disorder, pas de } \\
\text { diagnostic, etc. }\end{array}$ & 28 \\
\hline Total & & 63 \\
\hline
\end{tabular}

Source : dossiers d'interdiction, Saint-François, 1880-1920.

champ de connaissances qui n'est manifestement pas leur spécialité, peut de la sorte être examinée. Ces témoignages de médecins « ordinaires » sont tout à fait inégaux en termes de détails donnés. Il y a des attestations on ne peut plus succinctes d'aliénation mentale, comme dans le cas de Marceline B. : "après examen de Madame Marceline [B.], je constate qu'elle souffre d'aliénation mentale. En foi de quoi, je signe. J. A. Allard M.D. ${ }^{96} »$ Mais quand les dépositions des praticiens mentionnent des données cliniques, les constats effec-

96. CSS, FTC, n $^{\circ}$ 1992, 22 février 1905, interdiction et curatelle de Marceline B. 
TABLEAU 6

DIAGNOSTICS DES MÉDECINS NON SPÉCIALISTES

\begin{tabular}{|c|c|c|}
\hline & Diagnostics et exemples & Nombre \\
\hline Imbécilité & $\begin{array}{l}\text { Imbecile } \\
\text { Congenitally imbecile } \\
\text { State of total imbecility (second childhood) } \\
\text { Imbecility chiefly intellectual but to a certain extent also } \\
\text { moral. Moral imbecility avec kleptomania et dipsomania } \\
\text { (même cas) }\end{array}$ & $\begin{array}{l}2 \\
1 \\
1\end{array}$ \\
\hline $\begin{array}{l}\text { Softening of } \\
\text { the brain }\end{array}$ & $\begin{array}{l}\text { Softening of the brain } \\
\text { Chronic softening of the brain } \\
\text { Softening of the brain tissue, which has rendered him imbecile }\end{array}$ & $\begin{array}{l}3 \\
1 \\
1\end{array}$ \\
\hline Épilepsie & $\begin{array}{l}\text { Épileptique depuis l'enfance } \\
\text { Faible d'esprit par suite d'attaques d'épilepsie }\end{array}$ & \\
\hline Sénilité & $\begin{array}{l}\text { Senile atrophy } \\
\text { Progressive senility }\end{array}$ & \\
\hline Autres & $\begin{array}{l}\text { Mania } \\
\text { Arterio-sclerosis (suffering from a form of imbecility or } \\
\text { insanity known as...)* } \\
\text { Paralytic stroke (a slight) apparently affecting her brain } \\
\text { Paranoia or fixed idea [...] a progressive trouble } \\
\text { General breaking down from advanced age }\end{array}$ & $\begin{array}{l}1 \\
1 \\
1 \\
1\end{array}$ \\
\hline Imprécis & $\begin{array}{l}\text { Aliéné, aliénation mentale, esprit très faible, insane, feeble } \\
\text { condition of his intellect, unsound mind, weak intellect, etc. }\end{array}$ & 26 \\
\hline Total & & 45 \\
\hline
\end{tabular}

Source : dossiers d'interdiction, Saint-François, 1880-1920.

* S'agit-il d'une erreur du médecin?

tués ressemblent très fréquemment aux critères populaires de définition de la folie. Ce fait est absolument essentiel. Quand on demande à Léonidas-Charles Bachand, médecin du village de Coaticook, d'indiquer si un intimé est aliéné et de relever quelques comportements étayant son opinion, il répond que l'intelligence du défendeur s'est « affaiblie », que son raisonnement a changé, qu'il est adepte d'expéditions nocturnes impromptues et qu'il se livre à des spéculations hasardeuses. Bachand conclut que le défendeur est d'un " esprit très faible » et qu'il peut parfois « perdre contrôle sur lui-même et devenir 
furieux ${ }^{97} »$. Tout cela peut aussi bien être dit par un témoin du peuple. Très rares sont les dossiers où l'on se donne la peine de donner une apparence scientifique au discours tenu.

Un diagnostic aussi bien étayé que celui formulé par le docteur W. W. Lynch, dans le cas de William F., est exceptionnel. Selon Lynch, William F., un suicidaire, souffre d'une forme d'aliénation connue sous le nom de paranoïa ou idée fixe, un trouble progressif et souvent associé à des tendances maniaques, c'est-à-dire une tendance à la destruction de sa propre existence ou celle d'autres personnes. À certains moments, le sujet est assez lucide. Un changement peut survenir n'importe quand dans sa condition mentale, pour le meilleur ou pour le pire. Le médecin termine en disant que c'est tout ce qu'il peut dire sans entrer dans les détails ${ }^{98}$. Des cultivateurs ne sont de toute évidence pas en mesure de tenir un tel discours.

En d'autres occasions, lorsqu'un trouble précis est identifié et que l'auteur du verdict s'explique, cette impression d'expertise en aliénation disparaît. George M., d'après son médecin, a commencé huit ans plus tôt à montrer des signes de "softening of the brain », et celui qui le soigne ne le considère pas sain d'esprit depuis ce temps. Voici les indices de dérangement relevés par le praticien : l'homme en conversant change abruptement de sujet, parle d'une façon irrationnelle et fait des affirmations absurdes et déraisonnables ${ }^{99}$. Parents et amis, encore une fois, sans faire mention de ramollissement du cerveau, peuvent rapporter exactement les mêmes observations. Un autre constat surprenant est celui de l'absence presque complète de tentatives d'explication organiciste de l'aliénation mentale ${ }^{100}$. Seules deux mentions de tendances héréditaires ou familiales ont été retrouvées ${ }^{101}$ et on ne peut clairement percevoir un effort fait pour relier un état mental précis à des symptômes physiologiques très clairs que dans un seul cas ${ }^{102}$.

Une déposition affecte même une forte dose de moralisme. Charles J. Edgar de North Hatley constate chez Edward C., selon ses termes, une imbécillité d'une nature intellectuelle mais aussi, dans une certaine mesure, morale. Il invoque d'abord l'absence d'éducation de l'intimé, bien que celui-ci ait passé plusieurs années à l'école. Ensuite, apprenons-nous, l'imbécillité morale se manifeste sous deux formes, soit la kleptomanie et

97. CSS, fonds testament, boîte cs $4, \mathrm{n}^{\circ} 320,12$ juillet 1882 , nomination d'un conseiller judiciaire à Eli E.

98. CSS, FTC, $\mathrm{n}^{\circ}$ 4012, 22 août 1918, interdiction et curatelle de William F.

99. CSS, FTC, $\mathrm{n}^{\circ} 326,17$ décembre 1888 , interdiction et curatelle de George M.

100. Il faut noter que la doctrine de la dégénérescence est organiciste. Voir G. Grenier, op. cit., 24.

101. CSS, FTC, $\mathrm{n}^{\circ} 1281,25$ novembre 1898 , interdiction et curatelle de Samuel D. et CSS, FTC, $\mathrm{n}^{\circ} 1269,3$ octobre 1898 , interdiction et curatelle d'Isabella M.

102. CSS, FTC, $\mathrm{n}^{\circ} 2582,11$ octobre 1909 , interdiction et curatelle de Frederick T. 
la dipsomanie. Le médecin a eu connaissance de démonstrations fréquentes des deux types chez le défendeur. En commettant des infractions de ce genre, l'intimé n'a pas fait preuve de regrets. En fait, son imbécillité n'est pas d'une forme très sévère. Il dépense de l'argent, boit, est frivole et ses capacités mentales sont faibles ${ }^{103}$. Des normes culturelles propres à une époque ont de toute évidence joué un rôle central dans le sort de l'accusé, surtout si on se rappelle l'importance de la représentation de l'ivrognerie comme fléau social dans les conceptions du tournant du siècle.

La lecture et l'analyse des témoignages de médecins non spécialistes laissent perplexe. S'ils ne sont pas tenus à des interventions détaillées, la ressemblance très fréquente de leurs dépositions avec les dires des gens ordinaires ${ }^{104}$, la rareté d'explications organicistes de la folie ou de considérations étiologiques peut nous conduire, à cent ans de distance, à émettre l'interrogation suivante : ces praticiens, probablement dépourvus d'expérience professionnelle sérieuse au chapitre de l'aliénation, s'expriment-ils seulement en vertu de leur autorité? Leur présence dans les procès ne relève-t-elle que d'un certain prestige scientifique? Il semble bien que le concours des médecins n'a rien d'essentiel. Aucun praticien ne s'oppose aux accusations de folie. Ils ne feraient conséquemment que cautionner un processus en cours où le constat populaire serait peut-être véritablement le maître. Les certificats d'aliénistes, pour leur part, représentent la consécration officielle de l'état de personnes déjà internées.

\section{LE RECOURS À L'ASILE}

C'est le lot de la moitié des individus du corpus, car 69 personnes sur 138 se trouvent à l'asile au moment de la présentation d'une requête visant à les interdire ${ }^{105}$. La période étudiée connaît au surplus une accélération du recours à l'enfermement. De 1880 à 1900 inclusivement, 16 personnes en passe d'être interdites sont internées, ce qui représente $32 \%$ des 50 aliénés interdits à ce moment dans le district judiciaire de Saint-François, contre 53 de 1901 à 1920 inclusivement, ce qui constitue $60,2 \%$ des 88 personnes connaissant la même situation au cours de cette deuxième période. L'exclusion physique du fou derrière les portes closes de Verdun, Beauport et Saint-Jean-de-Dieu prendrait de l'ampleur après

103. CSS, FTC, $\mathrm{n}^{\circ}$ 1286, 25 novembre 1898, interdiction et curatelle d'Edward C.

104. Scull a souligné les parallèles entre les définitions médicales de la folie et les conceptions populaires de celle-ci en Angleterre au XIX ${ }^{\mathrm{e}}$ siècle. A. T. Scull, op. cit., 239.

105. Pour d'utiles perspectives sur les liens entre situations familiales et internements asilaires, on consultera D. Wright, « Getting Out of the Asylum : Understanding the Confinement of the Insane in the Nineteenth Century », Social History of Medicine, 10,1 (avril 1997) : 137-155. 
la phase de mise en place des institutions asilaires québécoises au XIX siècle. Le secours de l'asile semble entrer dans les mœurs, ce qui lui assure de facto une certaine "présence » dans la communauté.

Parallèle intéressant, les interdits envoyés à l'asile affichent les mêmes caractéristiques générales que celles du groupe entier d'interdits. Du point de vue des sexes, le partage des internés équivaut à celui rencontré dans l'ensemble de notre population de malades $(75,4 \%$ d'hommes contre $24,6 \%$ de femmes). Quant au clivage linguistique, la situation paraît à son tour sensiblement la même que dans la totalité des documents étudiés (62,3\% d'anglophones contre $37,7 \%$ de francophones). Il n'y a pas non plus de différence entre les interdits et le sous-ensemble des internés au chapitre de l'habitat $\left(81,2 \%\right.$ de campagnards et $15,9 \%$ de citadins $\left.{ }^{106}\right)$. On peut toutefois présenter les caractéristiques des internés d'une autre façon que par le rapport à la totalité de ce sous-groupe. En effet, 53,1\% des hommes interdits (52 sur 98) sont déjà internés, contre 42,5\% des femmes interdites (17 sur 40). 49,1\% des campagnards interdits (56 sur 114) se trouvent déjà à l'asile, contre $61,1 \%$ des citadins interdits (11 sur 18), ce qui accréditerait la thèse faisant de l'urbanisation une des conditions d'une intolérance plus grande à la folie ${ }^{107}$, mais vu le faible nombre d'habitants des villes dans le corpus, l'affirmation demeure très risquée dans le cas présent. Enfin, 45,3\% des anglophones interdits (43 sur 95) et 60,5\% des francophones interdits ( 26 sur 43 ) ont été préalablement placés en institution : en somme, si les gens de langue française ont moins recours à l'interdiction, les placements à l'asile des futurs interdits seraient peut-être proportionnellement plus élevés chez eux. Les différences ethniques et culturelles dans le règlement de la folie demandent encore bien des recherches.

\section{CONCLUSION : DES AFFAIRES DE FAMILLE}

Les dossiers d'interdiction pour folie du tournant du siècle représentent en somme une scène où s'agitent différents agents de la question de l'aliénation mentale : le défendeur lui-même, son entourage, la justice, la médecine et l'asile. L'interdiction est un carrefour et, de ce fait, permet d'appréhender le jeu et les rapports de force entre les différents acteurs s'activant autour d'un même « problème », la présence du malade mental. Les thèses classiques de la montée du contrôle social de la folie au $\mathrm{XIX}^{\mathrm{e}}$ siècle, mainmise que des élites médicales et étatiques ont assurée par des moyens essentiellement institutionnels, méritent d'être revues et

106. Le lieu de résidence est inconnu dans deux cas.

107. Voir supra, note 48. 
complétées par des analyses de terrain plus fines. Le chercheur découvre alors des réalités s'écartant sensiblement de ce schéma.

Au-delà du fait que le Code civil accorde explicitement un rôle prépondérant aux parents et alliés, l'examen des dossiers d'interdiction montre que l'instance familiale et communautaire joue réellement un rôle de premier plan dans la gestion de la folie au tournant du siècle et ce, même après le développement d'un réseau d'institutions asilaires auxquelles on fera, tout de même, de plus en plus appel. L'examen des stratégies adoptées lorsque survient cette forme de déviance permet de redonner une place aux proches, aux parents, au voisinage. Le lieu du constat des manifestations de folie et de leur prise en charge temporaire est de toute évidence la maisonnée. Aussi, l'importance essentielle des questions de patrimoine, que signalent la forte proportion d'hommes interdits et les dénonciations des requêtes, de même que les spécificités sociologiques des individus traduits en justice (différences selon la langue, variété des occupations), laissent entrevoir l'action de facteurs qu'une perspective de contrôle social des masses pauvres par les élites ne peut saisir. Par ailleurs, à notre avis, les élites médicales régionales n'ont pris part qu'à titre de figurantes dans ces procès, procès amorcés la plupart du temps en raison des préoccupations normatives de l'entourage des malades. La variable de l'urbanisation-industrialisation, enfin, ne paraît pas avoir conditionné la sanction de l'aliénation dans le district judiciaire de Saint-François. Pour toutes ces raisons, une histoire sociale étudiant simultanément plusieurs pans de la logique d'ensemble d'une époque dans son règlement de la question des troubles mentaux laisse entrevoir ses possibilités. 